\title{
Linx
}

Revue des linguistes de l'université Paris X Nanterre

$7 \mid 1995$

Saussure aujourd'hui

\section{L'ambivalence théorique dans la recherche saussurienne sur la légende et les Notes item}

Francis-Marie Gandon

\section{OpenEdition}

Journals

Édition électronique

URL : http://journals.openedition.org/linx/1154

DOI : 10.4000/linx.1154

ISSN : 2118-9692

Éditeur

Presses universitaires de Paris Nanterre

Édition imprimée

Date de publication : 1 septembre 1995

Pagination : 201-217

ISSN : 0246-8743

\section{Référence électronique}

Francis-Marie Gandon, « L'ambivalence théorique dans la recherche saussurienne sur la légende et les Notes item », Linx [En ligne], 7 | 1995, mis en ligne le 13 juillet 2012, consulté le 20 avril 2019. URL http://journals.openedition.org/linx/1154; DOI : 10.4000/linx.1154

Ce document a été généré automatiquement le 20 avril 2019

Département de Sciences du langage, Université Paris Ouest 


\title{
L'ambivalence théorique dans la recherche saussurienne sur la légende et les Notes item
}

\author{
Francis-Marie Gandon
}

1 S'il est un champ que la thématique du double instruit et fissure de façon obsédante, c'est bien celui auquel Ferdinand de Saussure consacra une recherche polymorphe inaboutie et fascinante, il y a maintenant un siècle.

2 A cette perspective clivée, plus ou moins gommée dans l'édition du Cours, un premier responsable : Saussure, bien entendu, qui, dès 1894, écrivait :

«Nous nourrissons depuis bien des années cette conviction que la linguistique est une science double, et si essentiellement (barré) profondément, irrémédiablement double qu'on peut même (barré) à vrai dire se demander s'il y a une raison suffisante pour continuer à (barré) maintenir sous ce nom de linguistique une unité illusoire (barré) factice, génératrice précisément de tous les inextricables pièges contre lesquels nous nous débattons vainement (barré) chaque jour, avec le sentiment $»^{1}$

«...d'une totale impuissance» poursuit conjecturalement R. Engler ${ }^{2}$ dans une lecture estimée par Ch. Stetter comme tout-à-fait plausible ${ }^{3}$.

Le duel, ici sous la forme d'une opposition entre diachronie et synchronie, se donne donc dès l'origine comme interne à la linguistique qu'il empêche de se constituer en science véritable, tout en investissant son objet d'une charge passionnelle particulièrement lisible dans l'activité mythologique

5 Telle qu'elle ressort de l'analyse des Notes item $^{4}$ et de la légende germanique ${ }^{5}$, cette ambivalence prend différents visages : celui du signe, soumis à une relativité à la fois absolue (dissimile) et restreinte (simile), écartelé entre une double identité (synchronique et diachronique) ; celui d'une langue parfois pur système de différences, disposant parfois d'un substrat matériel, d'un support diachronique ; celui d'une diachronie souhaitée par moments systématique et constitutive, le plus souvent destructive et non réglable; celui des métaphores du signe (ou du symbole dans la légende), dont certaines - celle par 
exemple du «ballon » dans les Notes item - répudient toute tendance à la substance et à la totalisation, alors que d'autres - dans des passages parfois tout proches - reconduisent les platitudes les plus déprimantes (corps/âme, voix/esprit... ) ; celui des plans du signe, désirés et non désirés autonomes, analysables et non analysables, dans un contexte théorique qui parfois préfigure Hjelmslev et parfois s'en éloigne à outrance...

Il s'ensuit que la définition du signe est, dans un premier temps en suspens, celle de ses plans présentant, dans un second temps, un caractère problématique analogue. Nous envisagerons l'une et l'autre composantes de cette ambivalence théorique, sans toutefois celer, dans une brève présentation, les effets de cette ambivalence sur le métadiscours : qui ne se rend compte à quel point, en effet, le discours critique sur Saussure est luimême clivé ?

\section{Le pluriel de l'oeuvre : l'ambivalence dans la critique}

7 L'ambivalence concerne ici le déni qu'on oppose à la possibilité d'un regard unitaire (et d'outils conceptuels homogènes) sur une pluralité de champs qui, même si traités parallèlement, sont apparus successivement : recherches sur les Nibelungen, à partir d'un cours professé pour la première fois pendant, l'été $1904^{6}$; travaux sur les Anagrammes commencés, selon certaines sources, pendant le voyage en Italie de 1906 ; la réflexion sur la linguistique générale, indépendamment des cours donnés, est quant à elle, largement antérieure à 1894 si l'on en croit le passage cité plus haut ${ }^{7}$.

On peut dans ce cadre suivre avec curiosité le développement d'un discours de type docteur Jekyll/Mr Hyde, qui oppose le «visage nocturne de Ferdinand» au professeur genevois (pour emprunter à l'ironie d'H. Parret) ou encore l'«herméneute» à l'universitaire, selon le clivage de M. Pêcheux et F. Gadet, qui voient à l'oeuvre une " schizophrénie » nocturne défaisant le travail des dichotomies diurnes »".

Dans la lignée des « deux Saussure » (Recherches, 1974), L.J. Calvet parle d'une « véritable révolution » à propos des anagrammes ${ }^{9}$.

Pour M. Arrivé, le discours saussurien sur le symbole se construit de façon « autodestructive $»^{10}$, tandis que Sungdo Kim s'interroge : « Le mythologue Saussure est-il encore Saussure? ? (art. cit.).

11 Le plus curieux est que les points de vue inverses qui se représentent une méthodologie au fond homogène portant sur des objets divers, loin de rassurer, apportent un trouble supplémentaire. Ainsi en va-t-il de la suggestion d'o. Ducrot quant à l'explication de la présence régulière de la voyelle dans la syllabe - et à partir de là des régularités anagrammatiques - par une possible fonction d'ordre polémique aujourd'hui oubliée ${ }^{11}$ : s'ouvre une faille où la linguistique comme science de la Forme risque de disparaître. De même quand Avalle indique que "(les) théories de Saussure sur la légende ne diffèrent pas de ce que nous trouvons dans le CLG $»^{12}$ il propose un paradoxe rassurant à propos duquel l'autocontestation joue à fond puisqu'il est immédiatement anéanti par ce qui suit : la nouveauté totale introduite « dans la doctrine du signe (ou symbole) » (ibid.).

Nouveauté d'ailleurs niée par T. Todorov qui affirme l'univocité théorique du symbole: «Dans les études sur les Nibelungen (Saussure) ne reconnaît des symboles que pour les attribuer à des lectures erronées $»^{13}$. Comme on le sait depuis la publication des manuscrits c'est évidemment ici la lecture de Todorov qui est erronée. 
$13 \mathrm{Au}$ delà de la problématique d'un champ sémiolinguistique relativement homogène ou irrémédiablement fissuré nous souhaiterions insister sur le fait que l'ambivalence travaille le dispositif théorique lui-même ce dont témoigne une création constamment renouvelée d'analogies qui s'efforce à une impossible suture. Parmi "toutes (ces) analogies du ciel et de la terre» l'une particulièrement symptomatique : celle du jeu d'échecs, qui veut exprimer l'ignorance dans laquelle se tiennent mutuellement les composantes diachronique et synchronique de la langue : la suite des coups et la position. Or il a été curieusement peu remarqué que, fausse à plusieurs égards ${ }^{14}$, la métaphore l'est radicalement en ce qu'elle exclut le caractère polémique et stratégique du jeu. Caractère polémique nié : il n'est pas fait mention d'une partie d'échecs - c'est-à-dire d'un partage. Quant à la négation de son caractère stratégique, elle va jusqu'au paradoxe :

« (rien n'empêche, pour introduire dans la <comparaison> un trait assez essentiel, de supposer le joueur tout-à-fait absurde et inintelligent comme l'est le hasard des

événements phonétiques et autres [signe inintelligible] )» (N. Whit., fo 10)

L'image absurde d'un joueur supposé inepte ne sera plus présentée dans le Cours que comme une hypothèse nécessaire à la conformité de la comparaison.

Mais l'absurdité se propage à la métaphore elle-même, dont on ne peut pas ne pas relever le caractère autodestructeur : destinée à illustrer le double elle en forclot simultanément la pensée. L'ambivalence théorique en ce sens se conteste elle-même: de fait par un mouvement assez fréquent de la pensée de Saussure on s'aperçoit que des oppositions présentées comme essentielles ici ne donnent plus lieu ailleurs qu'à des disjonctions inclusives. Ces paradigmes glissants relèvent donc d'une contradiction au second degré, qui caractérise l'ambivalence théorique du maître de Genève.

\section{L'ambivalence interne : institution et dissolution du signe}

L'ample anaphore que constituent les Notes item ${ }^{15}$ semble contenir le stade le plus avancé de la pensée saussurienne vis-à-vis du signe. La plupart du temps ce dernier est mise en relation non plus comme dans la troisième version du Cours - d'un signifié et d'un signifiant, mais d'une signification avec un signifiant : plus précisément sème ou sôme, ce qu'a souligné par exemple C. Stetter ${ }^{16}$.

17 Certains passages rejettent de façon radicale la possibilité même du signe isolé et il n'est pas indifférent de voir évoquée la problématique du prédicat et de la phrase ${ }^{17}$ en même temps que la terminologie substitue au signe un "parasème " comme "signe faisant partie d'un système » ( $\left.f^{\circ} 5\right)$. Par delà le conventionnalisme à la Whitney, et en harmonie avec l'arbitraire radical du troisième cours ce dont la représentation est tentée - et Saussure se livre ici à un effort de définition sans précédent - est une entité s'anéantissant dès qu'abstraite d'un ensemble. De quelle nature est cet ensemble? Le passage suivant l'éclaire :

«Tous [les psychologues anciens ou modernes FG-] sans exception se figurent la langue comme une forme fixe, et tous sans exception comme une forme conventionnelle. Ils se meuvent, très naturellement dans ce que j'appelle la tranche horizontale de la langue, mais sans la moindre idée du phénomène social (barré) socio-historique qui entraîne immédiatement (barré) le tourbillon des signes dans la colonne verticale et défend alors d'en faire ni un phénomène (barré) langage fixe 
ni un langage conventionnel puisqu'il est le résultat incessant de l'action sociale imposé hors de tout [choix ?] ( $\left.f^{\circ} 3\right)$.

La biffure, qui substitue "socio-historique " à « social ", précise qu'est convoquée une diachronie «lourde » comme trait définitoire du système. La théorie conjugue donc ici arbitraire radical et histoire. D'une part en effet la langue se présente comme s'instituant de chaque acte de sémiose - récréation perpétuelle - mais la sémiose elle-même récapitule d'autre part une double dimension, synchronique et diachronique, et ne se contente plus de répartir des valeurs comme le donne à lire une certaine doxa saussurienne : ainsi dans l'analyse légendaire où la "masse sociale " "fixe à chaque instant la valeur [du symbole FG.] » (N-lég., 3958, IV, fo l).

La sémiose va bien sûr à l'encontre de la convention, qui suppose des termes déjà identifiés ${ }^{18}$ et l'existence d'une langue dans laquelle elle se fait ${ }^{19}$ - mais l'histoire ruine aussi cette notion, cette fois de façon plus naïve, plus "substantielle», puisqu'elle implique, du moins selon notre lecture, une certaine téléonomie, une Régularité : ce qui permet par exemple au symbole légendaire de varier « dans de certaines limites» (ibid.). D'où cette rencontre extrêmement troublante, de l'arbitraire radical et d'une certaine " motivation » associés à ruiner tout conventionnalisme.

On trouve dans le même passage un schéma représentant quatre cercles placés horizontalement et, sous le premier, deux autres à la verticale. Le cercle initial représente très vraisemblablement un signe comme négativement solidaire d'autres signes (en synchronie) tout en étant constamment travaillé (en diachronie) par l'évolution de la forme (évolution qui est aussi celle - mais dans quelle proportion ? - du sens).

"répulsive » et une lignée « évolutive »

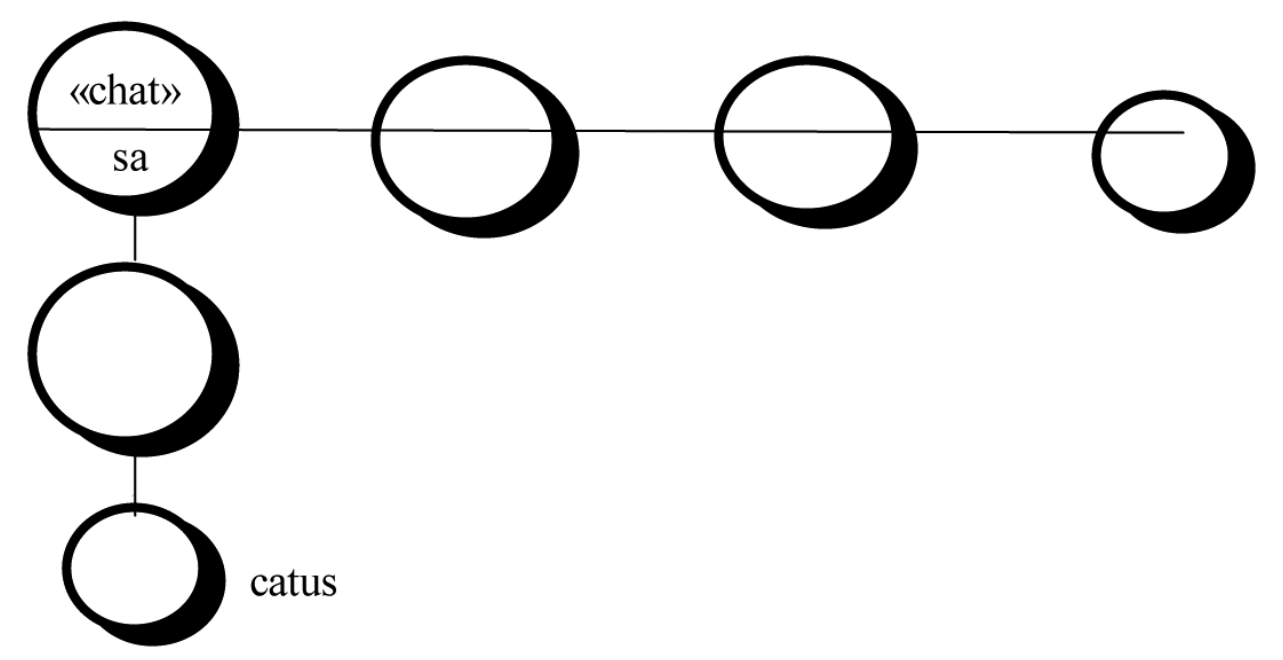

Fait surprenant l'évolution diachronique semble ici relever d'une Régularité :

« Non seulement il faut que les éléments [des formes nouvelles F.G.] soient puisées dans des combinaisons déjà fàmil[ières] (barré) connues, mais que tout se trouve pour ainsi dire préparé pour faire jaillir la combinaison nouvelle. » (N. It., $f^{\circ} 4 \mathrm{v}$ ).

Or de façon répétitive, tant dans le Cours que dans les sources manuscrites, seule la création analogique était digne d'une rationalité corrigeant précisément la nature fortuite du changement phonétique. Cette rationalité semble bien ici, sinon élargie à la diachronie dans son ensemble, au moins excéder le domaine de la création analogique. L'unité linguistique n'apparaît donc plus que comme le point d'intersection d'une double 
systématicité : une systématicité synchronique et ce que Saussure suggère relever aussi, au plan diachronique, du systématique.

Mais c'est une suggestion contestée quasi immédiatement puisque les « lois " phonétiques n'ont « aucun droit à ce nom ».20 (N. it. $\left.\mathrm{F}^{\circ} 5\right)$.

Tout en évitant tout retour à un positivisme néo-granmairien, Saussure laisse ainsi le lecteur des Notes dans une douloureuse incertitude.

Même incertitude dans certains passages des Nibelungen:

« Au bout de quelque temps..[la rune Y F.G.] est la dixième del'alphabet... mais ici déjà ELLE commence à supposer unité : que

Où est maintenant l'identité? On répond en général par sourire comme sans (barrés) (en marge: si c'était une chose en effet curieuse) remarquer la portée philosophique de la chose, qui ne va à rien moins que de dire que tout symbole, une fois lancé dans la circulation - or aucun symbole n'existe que parce qu'il est lancé dans la circulation - est à l'instant même dans l'incapacité absolue de dire en quoi consistera son identité à l'instant suivant. » (N. Lég. 3958, 4, fo 1).

Ici le changement diachronique provoque l'errance et l'émiettement de l'unité : aucune Régularité n'est plus opportune (alors que d'autres passages formulent, comme on le sait, des « règles » de type : déplacement, condensation, loi de non « dépoétisation », oubli et absence de mention comme facteurs positifs, résistance du non propre, caractère aposteriorique des interprétations mythiques ou religieuses - contrairement à W. Propp...).

Dans les Notes item un effort terminologique remarquable contraste avec la base matérielle de la métaphore privilégiée : celle du ballon. Le ballon est le « sème total » ou " parasème ", dont l'enveloppe est l'« aposème " - ou "sôme " - comme "sphère toute physique ». La paronomase platonicienne surdétermine-t-elle cette terminologie? L'aposème est dit être le "corps» voire le «cadavre» du sème ( $f^{\circ}$ 9) et englobe un « aposème intellectuel » ou " contre-sôme ». On peut donc admirer le contraste entre une terminologie purement relationnelle (qui représente sans doute l'effort extrême de Saussure dans ce domaine) et une métaphorique simpliste et quelque peu morbide... Comme on le voit une double lignée se déploie : le «signifié» du troisième cours, par exemple, devient «aposème intellectuel » dans la première et dans la seconde contresôme ", "anti-sôme ", "parasôme " ${ }^{21}$. Ce relativisme axiomatique est en principe aux antipodes des images habituelles: feuille de papier, dedans/dehors, âme/corps, accouplement ${ }^{22} \ldots$ cependant jamais complètement répudiées : la note finale oppose par exemple formes «vocales» et "spirituelles» Il dénie même au signe une vertu totalisante : dans un passage à vrai dire obscur Saussure caractérise le «sème » et la « sphère du sème » comme situés entre sôme et contre-sôme (f॰ 17$)$. On pourrait comprendre qu'il s'agit d'un compromis entre l'illimité du contre-sôme et la limite du sôme ; en ce sens le signe n'est nulle part: il n'est ni l'enveloppe du ballon ni le gaz à l'intérieur - et pourtant l'essentiel est bien «le ballon» pour l'aérostier. Cet essentiel est donc d'une certaine façon une abstraction. Mais cette abstraction est agonistique, "dialectique»: c'est une tension. On voit à quel point les Notes item visent à rompre avec la conception statique et organiciste, du signe, sans d'ailleurs y parvenir vraiment. 


\section{L'ébauche d'une analyse en traits : tentations et rétractations}

l'hypothèse d'un dualisme radical signe/signification - dualisme qu'on ne trouve d'ailleurs jamais dans la vulgate du Cours, contrairement à une idée reçue. Dans les Notes item l'absence d'une totalité subsumante, donc l'autonomie accordée à chacun des plans, est au moins envisagée comme un possible théorique. La métaphore du ballon voudrait donc que seules importent l'enveloppe-signe et l'hydrogène-signification (signification valant ici pour «contenu») sans que le «ballon» soit rien pour sa part, bien qu'il soit «tout pour l'aérostier » $\left(f^{\circ} 17\right)$.

« On ne peut vraiment maîtriser le signe, le suivre, comme un ballon dans les airs, avec certitude de le rattraper que lorsqu'on s'est rendu complètement compte de sa nature nature double, ne consistant nullement dans l'enveloppe et pas davantage dans l'esprit, dans l'air hydrogène qu'on y insuffle et qui ne vaudrait rien du tout sans l'enveloppe. » ( $\left.f^{\circ} 17\right)$.

Pour jouer un rôle de subsomption quant à ses deux faces, le signe doit lui même être compris comme scindé : étrange paradoxe

B. L'analysabilité du signifiant est ce qui, en apparence présente le moins de difficultés. Cependant Saussure a) rejette la notion d'unités organiques (morphologiques); b) donne à 
la divisibilité du sôme une assise temporelle qu'il n'arrive pas réellement - et en dépit de plusieurs métaphores (spatiales : faut-il s'en étonner ?) à définir :

« Le sôme sera comme le cadavre divisible en parties organisées ce qui est faux.» ( $\mathrm{F}^{\circ}$

$14 \mathrm{v})$.

Mais dans cette question il faut donner grande attention au mérisme (à la divisibilité

dans le temps) des parties des mots. " (f॰ 16v). ${ }^{23}$

"Sémisme », « divisibilité par tranches », « transmission acoustique », " parole »... : notions précisant la linéarité du signifiant et opposant le signe linguistique « unispatial » au signe «multispatial » comme l'est un tableau allégorique. On voit par parenthèses se dessiner ici une esquisse de divergence radicale entre linguistique et sémiologie. La recolligibilité du sème est l'aspect complémentaire d'une divisibilité ailleurs affirmée comme le « fait le plus capital de la langue » ( $\left.f^{\circ} l 0\right)$. La nature temporelle de la recolligibilité est inaccessible à la conscience qui ne considère qu'une juxtaposition spatiale.

L'autonomie analytique du signifiant repose d'autre part sur une base physiologique :

«Lors même qu'on dépouille un signe de son son, toujours l'esprit met au moins dans le signe (ou le sôme) au moins L'INTENTION (de signifier) (barré), qui met les MUSCLES en mouvement et prouve ainsi une volonté. » $\left(\mathrm{F}^{\circ} 14 \mathrm{v}\right)$.

41 Ici la matière de l'expression, le «purport » de Hjelmslev s'esquisse mais ailleurs seule l'«idée » du mot permet de préciser des nuances de prononciation ( $f^{\circ} 22$ v). D'où la contradiction suivante : un signe sous abstraction du sens est envisageable / seul le sens permet de spécifier le signifiant dans ses nuances. On a évidemment relevé que la nouveauté théorique des Notes tient dans la composante diachronique du signe, "sème colligible quoique composé de parties successives linéaires » ( $\left.f^{\circ} 4\right)$, qui devient le lieu d’une conversion d'une diachronie "opérative» en une perception synthétique à son tour décomposable.

Touchant l'analysabilité du signifié les difficultés apparaissent comme sérieuses dans la mesure où Saussure dissocie soigneusement une "sémantique» éventuelle de la dimension génétique des mots. C'est ainsi que l'association d'un thème vocal à une « idée diffluente » (expression guillaumienne, anachronisme voulu) n'est pas considéré comme pertinent. A la parenté génétique est en effet opposé un « système psychologique » ( $\left.f^{\circ} 8\right)$ en fait un paradigme associatif - qui précise la notion de "signe en contexte» ou parasème :

Pour un mot quelconque faisant partie de la langue un second mot même n'ayant avec le premier aucune "parenté ». est un parasème. La seule et simple qualité du parasème est de faire partie du même système psychologique de signes. » ( $\left.f^{\circ} 8\right)$.

Ici le déni d'autonomie du signe n'est pas absolu - comme dans le Cours et d'autres passages des Notes - mais relatif à un code restreint substitué au système global; la possibilité d'un « mot autonome » est préservée

« [...] si l'on trouve, après observation, qu'un signe donné a sa complète existence hors des signes concurrents du système, le mot de parasème devra tombera » (ibid.). Interne au signe le mode d'appréhension du contenu ouvre la possibilité d'une analyse en figures. Mais par un nouveau renversement cette possibilité est théoriquement déniée :

«Ce qu'on appelle la signification est-ce que-nous appelons le parasôme, et, à la différence du sôme, ne peut jamais être dégagée de manière à devenir pour ellemême un objet de recherche ou d'observation. » ( $\left.f^{\circ} 17 \mathrm{v}\right)$.

Et

Il s'agit de limiter dans leur sphère respective le sôme et l'anti-sôme. Or le sôme se limite facilement dans sa sphère, qui est toute physique. C'est là l'origine et 
l'explication des orgies bouffonnesques. L'anti-sôme n'est pas du tout limitable dans sa sphère. » (ibid.).

particulier désigner comme «traits du contenu »: forme $\tau$, son 8è lettre de l'alphabet, nom mystique de zann, fait qu'elle soit parfois citée comme première du mot (?) (N. lég. 3958, IV, 1). Les critiques - Saussure lui-même - ont souligné la nature menacée d'une telle identité mais sans en voir l'aspect complémentaire un contenu dénombrable et nanti d'un rudiment d'organisation.

«C'est seule la durée relative de certains traits qui donne illusion là-dessus, et c'est la leçon de tous les jours pour qui étudie de voir que l'association - que nous chérissons parfois n'est qu'une bulle de savon, n'est pas même une bulle de savon, laquelle possède au moins son unité physique et mathématique " (N. lég.3958, VII, $21 \mathrm{v})$.

L'aspect complémentaire "positif » est en effet l'association d'une expression à un nombre restreint de traits du contenu. Nous sommes donc ici en dehors de la problématique du signe découpant la même mesure des deux plans signifié/signifiant (cf. l'image de la feuille de papier). Sans qu'on puisse bien entendu parler d'isomorphisme Saussure n'évoque à aucun moment une possible «organisation analogue » des deux plans - la non coïncidence entre les unités de l'un et l'autre plans en rend envisageable une formulation théorique ultérieure. 
51 Ainsi la dimension diachronique contribue-t-elle puissamment à stratifier le continuum amorphe du signifié (tout en fragilisant l'effet de cette stratification). Seules des réflexions à base diachronique (et plus précisément empruntant au caractère destructeur, héraclitéen, du temps) anticipent donc l'une des pointes du synchronisme : l'axiomatique hjelmslevienne. Troublant est à cet égard, sous l'égide de Von Humboldt, un rapprochement entre la formule saussurienne :

«Ce qui fait la noblesse de la légende comme de la langue, c'est que condamnées

l'une et l'autre à ne se servir que d'éléments apportés devant elles et d'un sens quelconque, elles les réunissent et en tirent continuellement un sens nouveau. « (N. lég. 3959, VII, 18).

et la définition du langage chez Hjelmslev

"Le langage est donc tel qu'à partir d'un nombre limité de figures qui peuvent toujours former de nouveaux arrangements, il puisse construire un nombre illimité de signes. $»^{24}$.

52 Ces deux textes se répondent, certes, mais comme le passionnel (noblesse, condamnées) au neutre; l'imprécis (éléments apportés) au précis. Un mouvement analogue de pensée se donne à lire, mais produisant là une formation du sens à partir du sens, ici l'arrangement du signe à partir du non-signe. La différence cardinale tient en ce que l'« élément » saussurien est d'essence diachronique. Or il n'est pas abusif de voir dans le temps un facteur ambivalent par excellence : engendreur et destructeur de signes. Dans cette optique le rapprochement entre les deux passages est merveilleusement inexact (je veux dire que la coïncidence tient autant du rêve que de la science).

Même pression du flux héraclitéen dans le destin, déjà illustré, des paradigmes. Autre exemple : l'une des Notes item précise l'absence de morphologie « hors du sens » - alors que la face symétrique du "sens » est l'aposème, constamment considéré par ailleurs comme indépendant de la morphologie. Ce caractère fugitif et glissant des paradigmes, les Notes sur Whitney en donneront un dernier cas (pendant de la séquence initiale de notre intervention) où l'« irritante duplicité du langage » trouve sa version soft : Saussure se contentant d'écrire qu'il n'y a "aucun danger " à insister surtout sur le côté non historique du langage » $(N$. Whit., $13 \mathrm{v})$. Une telle timidité non seulement stupéfie mais souffle en quelque sorte la charpente même de l'oeuvre: la contradiction majeure diachronie/ synchronie se dilue dans une sorte de recommandation morale où la rigueur scientifique (la loi d'airain du choix) s'abolit en un vel, un « si vous voulez » généralisé.

Identique trait destructeur du temps - c'en est même le leitmotiv dans la légende germanique avec cette précision que l'altération des éléments en constitue la valeur. La pertinence n'y existe en effet que dans le pâtiment: seule l'altération produit le symbole - et l'énigme ${ }^{25}$ - et le désir. L'énigme résolue, ne reste que le référent historique: disparaissent donc et le symbole et le désir. La quête saussurienne prend ainsi le trait paradoxal suivant: il faut chercher à ne pas trouver et vouloir ne pas résoudre le problème posé et par là le destin du symbole, dont la valeur ne tient qu'au mouvement de sa dérobade ${ }^{26}$.

\section{Ambivalence, identité, destin}

55 Je m'aperçois que des termes comme «dérobade» "glissement» sont venus spontanément sous ma plume. 
56 Et puisqu'il faut savoir terminer une communication, pourquoi ne pas considérer ces deux lexèmes comme emblématiques d'une clôture - ou du moins de son semblant ? La dérobade du symbole renvoie à celle du signe, écartelé entre signification et valeur.

Le signe est en effet d'une part signification : processus d'appropriation opérative fondé sur une modalité brève de diachronie : cette (re)colligibilité est certes conditionnée par la linéarité (qui fait de la langue un objet sémiologique à part) mais contredit en même temps cette dernière. Opération mentale productrice de la conscience du signe cette (re)colligibilité n'a rien à voir avec la divisibilité morphologique - dissection d'un cadavre ${ }^{27}$ - même s'il est impossible de se la représenter autrement qu'en termes spatiaux ${ }^{28}$.

La substance. se voit donc renvoyée à l'histoire d'où la bifurcation entre les deux « voies du signe » : l'« historique » et la « consciente».

Outre la signification - processus interne, la valeur - processus latéral. Mais cette valeur elle-même se dédouble selon qu'elle est considérée comme relative au système ou au contexte sémantique local.

Vis-à-vis de ce dernier le sème est désigné comme parasème. Conséquence : seuls certains signes sont des parasèmes. D'où une double valence - générale et restreinte - de la valeur. D’où la dualité au second degré :

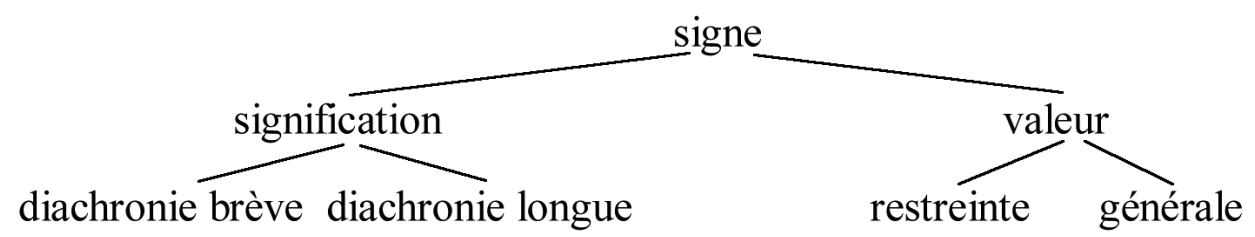

61 Mais précisément: ce dédoublement généralisé a pour effet de rendre irréels les paradigmes oppositionnels. Si l'on reprend les affirmations - quasi simultanées - qu'il n'y a de signe qu'en système ${ }^{29}$ et que certains sèmes peuvent avoir « leur complète existence hors les signes concurrents du système » ( $N$. it., fo 8 ), on constate un télescopage entre relativismes de rang diffèrent : en ce sens le dédoublement de la valeur ne dédouble rien mais souffle la notion de la relation même entre $\mathrm{S}$ et $7 \mathrm{~S}$ (non S).

Enfin - et ce sera ma dernière remarque - le «tourbillon » diachronique de la légende et la diachronie brève des Notes item doivent être considérés comme en relation de conflit ce qui accentue le caractère impossible de l'identité du signe..$^{30}$

63 A ce point où les dichotomies de la méthode s'entent à même la fissure de l'identité, chacun ressent que la problématique inscrite n'intéresse plus seulement la linguistique ou l'épistémologie, mais qu'elle touche à un trouble inscrit au coeur du destin humain, dans la mesure où tout destin est quête.

\section{NOTES}

1. Notes sur Whitney, $\mathrm{f}^{\circ} 14$, verso. Ces notes seront désormais citées par l'abréviation N.Whit.; l'indication du verso, comme pour les autres manuscrits de Saussure, sera désormais fournie par 
la lettre Y. Rédigées dans un cahier cartonné noir, les N.Whit. sont l'ébauche d'un article sur le linguiste américain, mort le 7 Juin 1894, et qui partage avec Saussure le trait contrasté d'une grande notoriété associée à une quasi-absence de publications (noté par Saussure). Pour la reproduction des manuscrits nous adoptons les conventions suivantes :

$<>$ texte restitué ; [ ] lecture conjecturale ; item (barré) : mot qui précède (ici item) barré dans le manuscrit.

2. Cours de linguistique générale, Wiesbaden, 1967.

3. "Linguistische Konsequenzen der Semiologie Saussures », Actes du Colloque Saussure d'Aix-laChapelle, 1983, in Zeichen und Verstehen, Aix-la-Chapelle, 1986, p. 49.

4. Les Notes item (désormais $N$. it.) sont, avec les N.Whit. et celles portant sur l'analyse de la légende germanique (désormais N.lég), à la Bibliothèque publique et universitaire de Genève, Salle Senébier. Elles comportent une vingtaine de feuillets.

5. Consacrées surtout au Nibelungenlied, chanson de geste d'auteur inconnu de la fin du XIIème siècle, les notes de Saussure se trouvent dans deux cartons; le premier (Ms fr. 3858) comporte huit cahiers (numérotés ici en chiffres romains), le second (Ms fr. 3959) dix cahiers plus deux enveloppes de notes, dont seule d'ailleurs la première intéresse la recherche sémiologique (même référenciation).

6. L'intérêt de Saussure pour l'épopée germanique remonte selon Møller à 1880 ; on sait qu'il assurera un cours de germanique à l'Ecole pratique des hautes études de 1881 à 1887 , cours qui s'élargira après cette date à d'autres langues et durera jusqu'en 1891. Pendant cette période Saussure semble avoir regretté de ne pas enseigner la littérature nordique.

7. Elle ne remonte donc pas à cette date comme l'indique Eugenio Coseriu (cité par Sungdo Kim in «Le mythologue Saussure est-il encore Saussure ", Linx n 22, Université de Paris X, 1990, p. 129).

8. La Langue introuvable, Maspéro, Paris, 1981. Curieusement Gadet de 1987 (Saussure, une science de la langue, P.U.F. Paris) ne mentionne pas Gadet de 1981: atteinte du méta-discours, dans son déploiement, par le clivage propre au texte-objet? Pour Pêcheux son discours saussurien fonctionnait dès 1969 (Analyse automatique du discours, Dunod, Paris) sur le mode science linguistique/résidu. Du moins à ce qu'affirme C. Normand (in «Langue/parole : constitution et enjeu d'une opposition ", Langages $n^{\circ} 49$, mars 1978, sous la direction de C. Normand, DidierLarousse, Paris, pp. 82-83).

9. Pour ou contre Saussure, Payot, Genève-Paris, 1975.

10. Linguistique et psychanalyse, Klincksieck, Paris, 1986, p. 38.

11. "Le structuralisme en linguistique » in Qu'est-ce que le structuralisme? », Seuil, Paris, 1968, rééd. « Points »,1973, pp. 106-107.

12. Avalle, d'Arco Silvio, «La sémiologie de la narrativité chez Saussure » in Essais de la théorie du texte, sous la direction de C. Bouazia, Galilée, Paris, 1973, p. 40.

13. Todorov, Tzvetan, Théories du symbole, Seuil, Paris, 1977, p. 337.

14. Autre trait non pertinent, déjà relevé : le «coup d'échecs » dans une partie réelle - doit tenir compte de la disposition de l'ensemble des pièces. Or un « coup » linguistique, telle l'alternance fot : foti, devenue fot : foet (manuscrits) ou gasti devenu gäste (Cours), ne peut, selon les thèses saussuriennes être mis en relation avec l'ensemble du système, d'une part à cause du caractère fortuit des mutations, de l'autre parce que cette relation élargie est l'apanage des " positions » en synchronie, c'est-à-dire des paires minimales (pour caractériser plus correctement les unités primaires: cf. C. Zilberberg, "Retour à Saussure?", Documents du Groupe de recherches sémiolinguistiques, VIII, 63, INALF, Paris, 1985, p. 18). Enfin - ne serait-ce qu'avec la création analogique - une "position» quelconque doit pouvoir garder la mémoire ou la trace d'un « coup » similaire effectué.

15. Texte peu facile à dater. Il y est fait allusion à la Sémantique de Bréal (1897) -- ce qui ne prouve pas grand chose. 
16. art.cit., pp. 40-41.

17. Amorce qui n'a rien de définitif : les notes finales opposent le signe - élément d'un « trésor » à la phrase en des termes qu'on retrouvera quasi littéralement sous la plume d'E. Benveniste («Sémiologie de la langue », Semiotica, Mouton, La Haye, 1969 et Problèmes de linguistique générale II, Gallimard, Paris, 1974).

18. T. de Mauro, édition du Cours de linguistique générale de Saumure, Payot, Genève-Paris, 1972, pp. 361-362.

19. C. Stetter, «La fonction des réflexions sémiologiques dans la fondation de la linguistique générale chez F. de Saussure ", Kodikas/Code, vol.1, n 1, janvier 1979, Gunter Narr Verlag, Tübingen, p. 15 .

20. Dans le Cours la question de l'identité diachronique reste, on le sait, irrésolue. Quant à la proposition de Hjelmslev: que deux états diachroniques d'une entité linguistique soient les « variants d'un invariant » elle ne permet pas de rendre compte de phénomènes comme le -per de « persécuter» ou de l'existence, dans «noueux» (exemple de Saussure), d'un radical et d'un suffixe etc.

21. A ce champ étymologique-paronomastique il faut ajouter l'iposème proposé par Lucidi pour désigner simplement l'unité saussurienne (cf. T. de Mauro, C.L.G., p. 458).

22. Cette dernière métaphore assez peu remarquée ; cf. toutefois Anne Hénault, « Perplexités à propos du terme complexe » Exigences et perspectives de la sémiotique. Recueil d'hommages pour A.J. Greimas, John Benjamins, Amsterdam-Philadelphie, 1985, t. I, p. 246.

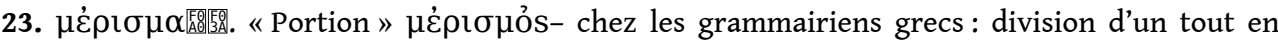
parties; d'un mot composé en ses éléments ; d'un vers en pieds. On sait l'emploi qu'en fera E. Benveniste pour désigner le trait pertinent («Tendances récentes en linguistique générale Journal de Psychologie, P.U.F., Paris, janvier-juin 1954, et « Les niveaux de l'analyse linguistique », Proceedings of the 9th International Congress of Linguists, Cambridge, Mass, 1962, Mouton \& Co. 1964, articles repris dans les Problèmes de linguistique générale I, Gallimard, Paris, 1966). Emploi d'ailleurs discret puisque limité - sauf oubli de notre part - à une seule occurrence dans chacun de ces articles (en ce qui concerne les Problèmes de linguistique générale).

24. Prolégomènes à une théorie du langage, 1943, tr. frçse, éd. de Minuit, Paris, 1968-1971, p. 64.

25. Dans l'analyse des Nibelungen- Saussure utilise assez fréquemment le terme de « rhapsodie », qui a le sens - on le sait - de « récitation » mais aussi de poème épique. Chez Sophocle le sphynx est désigné par l'expression de « chienne rhapsode » dans un passage d'Oedipe-roi.

26. cf. l'expression de « tourniquet masochiste » utilisée par M. Arrivé (op. cit., p. 36).

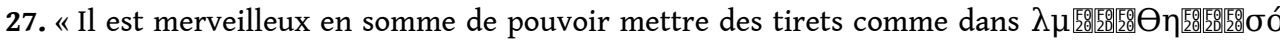
[F⿱ 20 [20] passif).

28. Cf. l'à peu près unique métaphore du disque de lanterne magique ( $f^{\circ} 12$ )

29. «Terme serait du reste assez <près de> ce que nous voulons dire par sème ; il y a quelque chose à remarquer à cet égard. Un synchronisme se compose d'un certain nombre de termes (termini) qui se partagent l'ensemble de la matière à signifier " ( $f^{\prime} 19$ v). On n'ignore pas le sens de « borne » ou de « limite » assigné au mot latin, et donc ici la conception purement relationnelle du sème.

30. Ainsi du signe linguistique : ailleurs considéré comme absolument spécifique (principe de linéarité), réduit dans telle note à la seule particularité de « produire une association plus précise que toute autre » (fo 18). Le paradigme linguistique/sémiologie est alors vidé de substance, comme disparaissent les notions de (re)colligibilité et de linéarité. 